\title{
A New Explicit Symplectic Fourier Pseudospectral Method for Klein-Gordon-Schrödinger Equation
}

\author{
Yanhong Yang ${ }^{1,2}$, Yongzhong Song ${ }^{1}$, Haochen $\mathrm{Li}^{3}$ \\ and Yushun Wang ${ }^{1, *}$ \\ 1 Jiangsu Key Laboratory for NSLSCS, Institute of Mathematics, School of Mathematical \\ Sciences, Nanjing Normal University, Nanjing 210023, China \\ 2 Department of Mathematics, College of Taizhou, Nanjing Normal University, \\ Taizhou 225300, China \\ ${ }^{3}$ LMAM, CAPT and School of Mathematical Sciences, Peking University, Beijing 100871, \\ China
}

Received 14 February 2017; Accepted (in revised version) 15 June 2017

\begin{abstract}
In this paper, we propose an explicit symplectic Fourier pseudospectral method for solving the Klein-Gordon-Schrödinger equation. The key idea is to rewrite the equation as an infinite-dimensional Hamiltonian system and discrete the system by using Fourier pseudospectral method in space and symplectic Euler method in time. After composing two different symplectic Euler methods for the ODEs resulted from semi-discretization in space, we get a new explicit scheme for the target equation which is of second order in space and spectral accuracy in time. The canonical Hamiltonian form of the resulted ODEs is presented and the new derived scheme is proved strictly to be symplectic. The new scheme is totally explicit whereas symplectic scheme are generally implicit or semi-implicit. Linear stability analysis is carried and a necessary Courant-Friedrichs-Lewy condition is given. The numerical results are reported to test the accuracy and efficiency of the proposed method in long-term computing.
\end{abstract}

AMS subject classifications: 65M70, 65M12, 65M06

Key words: Klein-Gordon-Schrödinger equation, Fourier pseudospectral method, symplectic scheme, explicit scheme.

\section{Introduction}

The Klein-Gordon-Schrödinger (KGS) equation

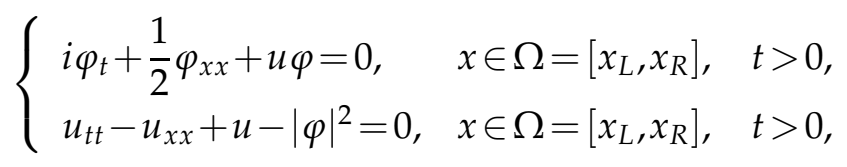

${ }^{*}$ Corresponding author.

Emails: yzynj@163.com (Y. H. Yang), yzsong@njnu.edu.cn (Y. Z. Song), wangyushun@njnu.edu.cn (Y. S. Wang) 
is a classical model used to describe a system of conserved scalar nucleons interacting with neutral scalar meson coupled through the Yukawa interaction in [6], where the complex-valued function $\varphi=\varphi(x, t)$ represents a scalar nucleon field, the real-valued function $u=u(x, t)$ represents a scalar meson field and $i=\sqrt{-1}$ (also see [14]).

Let $\varphi(x, t)=q(x, t)+i p(x, t)$, where $q(x, t)$ and $p(x, t)$ are real functions and let $u_{t}(x, t)=$ $2 v(x, t)$. The KGS equation (1.1) can be written as

$$
\left\{\begin{array}{l}
p_{t}=\frac{1}{2} q_{x x}+u q \\
q_{t}=-\frac{1}{2} p_{x x}-u p \\
v_{t}=-\frac{1}{2}\left(u_{x x}-u+p^{2}+q^{2}\right) \\
u_{t}=2 v
\end{array}\right.
$$

In this paper, we consider the periodic boundary conditions

$$
p(x+L, t)=p(x, t), \quad q(x+L, t)=q(x, t), \quad u(x+L, t)=u(x, t), \quad v(x+L, t)=v(x, t),
$$

and initial conditions

$$
p(x, 0)=p_{0}(x), \quad q(x, 0)=q_{0}(x), \quad u(x, 0)=u_{0}(x), \quad v(x, 0)=v_{0}(x),
$$

where $L=x_{R}-x_{L}$. Let $z=(p, v, q, u)^{T}$. Then (1.2) can be written as an infinite-dimensional Hamiltonian system

$$
\frac{d z}{d t}=J^{-1} \frac{\delta H(z)}{\delta z}
$$

with

$$
J=\left(\begin{array}{cc}
0 & I_{2} \\
-I_{2} & 0
\end{array}\right)
$$

$I_{2}$ is the identity matrix of dimension 2 and the Hamiltonian

$$
H(z)=\int_{x_{L}}^{x_{R}}\left[-\frac{1}{4}\left(p_{x}^{2}+q_{x}^{2}\right)+\frac{1}{4} u^{2}-\frac{1}{4} u_{x}^{2}+v^{2}-\frac{1}{2} u\left(p^{2}+q^{2}\right)\right] d x .
$$

The KGS equation posses the mass conservation law

$$
\mathcal{M}(t)=\int_{x_{L}}^{x_{R}}|\varphi|^{2} d x=\mathcal{M}(0)
$$

and the energy conservation law

$$
\mathcal{H}(t)=\int_{x_{L}}^{x_{R}} \frac{1}{4}\left(u^{2}+u_{t}^{2}+u_{x}^{2}+\left|\varphi_{x}\right|^{2}\right)-\frac{1}{2} u|\varphi|^{2} d x=\mathcal{H}(0) .
$$


Hamiltonian systems have been playing a fundamental role in many important fields including mechanics, astronomy, optics, meteorology, oceanography, electromagnetism, quantum field theory. Although the theory and algorithms have been well-developed for the Hamiltonian ordinary differential equations (ODEs), major challenges occur as the framework is extended for solving Hamiltonian partial differential equations (PDEs). This is due to the fact that the underlying phase space must be generalized from finite to infinite dimensions. Nevertheless, most of the existing discussions for treating Hamiltonian PDEs are based on methods of lines in which a PDE is first discretized in space to yield a large system of Hamiltonian ODEs $[13,15]$. The resulting ODEs are then integrated through a structure-preserving numerical method. The main difficulty in such approaches is how to ensure that the generated ODEs are Hamiltonian.

Symplectic geometric integrators have been very popular for the Hamiltonian system since the method was proposed by Feng in [5]. It have been proven to be robust, efficient and very accurate in preserving the long-time behavior of solutions of Hamiltonian systems [8]. The multi-symplectic methods that can preserve multi-symplectic conservation under appropriate discretization have attracted much attention in recent years [19]. Bridges in [2] proposed the multi-symplectic method to solve the Klein-Gordon equation. Reich in [16] proposed multi-symplectic Runge-Kutta scheme for the Hamiltonian wave equation. In [9] Hong et al. proposed the explicit multi-symplectic scheme to solve the nonlinear KGS equation. However as we known most of the symplectic schemes for the KGS equation are implicit or semi-implicit and require a lot of computation cost $[1,10-12,18,21]$. It is of great interest to solve the KGS equation by using a totally explicit scheme which can preserve the symplectic structure of the system exactly.

Spectral and pseudospectral methods are very important in the last decades because of their convergence accuracy in solving smooth problem [17]. Spectral or pseudospectral methods not only have high accuracy, but also can preserve the geometric structure of the original PDEs $[3,4]$. Therefore, it perform quite well in long time integration. That is why we use the Fourier pseudospectral method to semi-discrete the system.

In this paper, we use Fourier pseudospectral method in space direction and symplectic Euler method in time direction respectively for the Klein-Gordon-Schrödinger equation to obtain an explicit symplectic method of one order in time. Then, we use the composite technique for increasing the order of accuracy to derive an explicit symplectic scheme of second order in time and spectral accuracy in space. The advantage of the resulting full-discrete scheme is that it dose not need to solve any linear or nonlinear equation whereas the explicit scheme in [9] involves solving linear algebraic equation at each time level. We present the time cost in the simulation for the KGS equation. The result shows that the scheme computes faster than the explicit scheme in [9]. By applying linear stability analysis, a necessary Courant-Friedrichs-Lewy (CFL) condition which is often required for the explicit scheme is also given for our new scheme. The adjoint method of the symplectic scheme is briefly investigated.

The remainder of this paper is arranged as follows. In Section 2, the symplectic Euler Fourier pseudospectral method and the composition method for Klein-Gordon- 
Schrödinger equation are introduced and the main results are stated. In Section 3, structure-preserving properties and linear stability analysis of the new scheme are proposed. In Section 4, some numerical examples are reported to demonstrate the theoretical results. Finally we make conclusions.

\section{New explicit scheme of KGS equation}

\subsection{Semi-discretization in space}

In this section, we give a semi-discretization method by applying the Fourier pseudospectral method in space for the KGS equation. The main idea of the Fourier pseudospectral method is to approximate the partial differential operator $\frac{\partial^{2}}{\partial x^{2}}$ with the Fourier spectral differential matrix $D_{2}$.

Denote the spatial domain by $\Omega=\left[x_{L}, x_{R}\right]$ and $L=x_{R}-x_{L}$. We denote $x_{j}=x_{L}+j h$, $j=0,1, \cdots, N-1$ as the grid points and $h=L / N$ as the grid size. For even number $N$, we define $S_{N}=\operatorname{span}\left\{g_{j}(x) ; j=0,1, \cdots, N-1\right\}$ as the integration space, where $g_{j}(x)$ is a trigonometric polynomial of degree $N / 2$, and is given explicitly by

$$
g_{j}(x)=\frac{1}{N} \sum_{l=-\frac{N}{2}}^{\frac{N}{2}} \frac{1}{c_{l}} e^{i l \mu\left(x-x_{j}\right)}, \quad c_{l}= \begin{cases}1, & |l| \neq N / 2, \\ 2, & |l|=N / 2,\end{cases}
$$

with $\mu=2 \pi / L$.

We approximate $u(x, t)$ by $I_{N} u(x, t)$ which interpolates $u(x, t)$ at the collocation points

$$
I_{N} u(x, t)=\sum_{k=0}^{N-1} u_{k} g_{k}(x),
$$

where $u_{k}=u\left(x_{k}, t\right)$. By directly computing, we can easily verify that

$$
g_{k}\left(x_{j}\right)=\delta_{j k}= \begin{cases}1, & j=k, \\ 0, & j \neq k .\end{cases}
$$

The values of $\frac{\partial^{2}}{\partial x^{2}} I_{N} u(x, t)$ at the collocation points $x_{k}$ are obtained in terms of the value of $u_{k}$ and the Fourier differential matrix $D_{2}$, i.e.,

$$
\left.\frac{\partial^{2}}{\partial x^{2}} I_{N} u(x, t)\right|_{x=x_{k}}=\left(D_{2} U\right)_{k}
$$

where $U=\left(u_{0}, u_{1}, \cdots, u_{N-1}\right)^{T}$ and $D_{2}$ represents the second order Fourier differentiation matrix with the elements

$$
\left(D_{2}\right)_{j, k}= \begin{cases}\frac{1}{2} \mu^{2}(-1)^{j+k+1} \frac{1}{\sin ^{2}\left(\mu\left(x_{j}-x_{k}\right) / 2\right)}, & j \neq k, \\ -\mu^{2} \frac{2(N / 2)^{2}+1}{6}, & j=k .\end{cases}
$$

The following important lemma is proved by [4]. 
Lemma 2.1 (see [4]). For the matrix $D_{2}$ defined by (2.1), we have

$$
D_{2}=F^{-1} \Lambda_{2} F \text {, }
$$

where $F$ is discrete Fourier transform and $F^{-1}$ is the inverse discrete Fourier transform, which are defined by

$$
(F)_{j, k}=W_{N}^{-j k}, \quad\left(F^{-1}\right)_{j, k}=\frac{1}{N} W_{N}^{j k}, \quad W_{N}=e^{i \frac{2 \pi}{N}},
$$

and

$$
\begin{aligned}
& \Lambda_{2}=\operatorname{diag}\left(\lambda_{0}, \lambda_{1}, \cdots, \lambda_{N-1}\right), \\
& \lambda_{k}= \begin{cases}-(k \mu)^{2}, & k=0,1, \cdots, N / 2, \\
-[(k-N) \mu]^{2}, & k=N / 2+1, \cdots, N-1 .\end{cases}
\end{aligned}
$$

Remark 2.1. The property of the second order Fourier differentiation matrix $D_{2}$ plays an important role in the linear stability analysis for the symplectic Fourier pseudospectral method in Section 3.

Applying the Fourier pseudospectral method in space to the KGS equation (1.2) we obtain

$$
\begin{aligned}
& {\left.\left[\left(I_{N} p(x, t)\right)_{t}\right]\right|_{x=x_{k}}=\left.\left[\frac{1}{2}\left(I_{N} q(x, t)\right)_{x x}+\left(I_{N} u(x, t)\right)\left(I_{N} q(x, t)\right)\right]\right|_{x=x_{k}},} \\
& {\left.\left[\left(I_{N} q(x, t)\right)_{t}\right]\right|_{x=x_{k}}=\left.\left[-\frac{1}{2}\left(I_{N} p(x, t)\right)_{x x}-\left(I_{N} u(x, t)\right)\left(I_{N} p(x, t)\right)\right]\right|_{x=x_{k}},} \\
& {\left.\left[\left(I_{N} v(x, t)\right)_{t}\right]\right|_{x=x_{k}}=\left.\left[-\frac{1}{2}\left(\left(I_{N} u(x, t)\right)_{x x}-I_{N} u(x, t)+\left(I_{N} p(x, t)\right)^{2}+\left(I_{N} q(x, t)\right)^{2}\right)\right]\right|_{x=x_{k}},} \\
& {\left[\left(I_{N} u(x, t)\right)_{t}\right]_{x=x_{k}}=\left.\left[2\left(I_{N} v(x, t)\right)\right]\right|_{x=x_{k}} .}
\end{aligned}
$$

By using the notations

$$
\begin{array}{ll}
U=\left(u_{0}, u_{1}, \cdots, u_{N-1}\right)^{T}, & V=\left(v_{0}, v_{1}, \cdots, v_{N-1}\right)^{T}, \\
P=\left(p_{0}, p_{1}, \cdots, p_{N-1}\right)^{T}, & Q=\left(q_{0}, q_{1}, \cdots, q_{N-1}\right)^{T},
\end{array}
$$

we can get a semi-discrete system

$$
\left\{\begin{array}{l}
Q_{t}=-\frac{1}{2} D_{2} P-U \cdot P \\
U_{t}=2 V \\
-P_{t}=-\frac{1}{2} D_{2} Q-U \cdot Q, \\
-V_{t}=-\frac{1}{2}\left(D_{2} U-U+P^{.2}+Q^{\cdot 2}\right),
\end{array}\right.
$$


where $U \cdot P=\left(u_{0} p_{0}, u_{1} p_{1}, \cdots, u_{N-1} p_{N-1}\right)^{T}$ denotes point multiplication between vectors, $P^{2}=P \cdot P$.

Therefore (2.2) can be written as a finite-dimensional Hamiltonian system

$$
\frac{d Z}{d t}=J_{2 N}^{-1} \nabla H(Z)
$$

with the Hamiltonian

$$
H(Z)=-\frac{1}{4}\left(P^{T} D_{2} P+Q^{T} D_{2} Q+U^{T} D_{2} U\right)+\frac{1}{4} U^{T} U+V^{T} V-\frac{1}{2} \sum_{j=0}^{N-1}\left(p_{j}^{2}+q_{j}^{2}\right) u_{j},
$$

where $Z=\left(P^{T}, V^{T}, Q^{T}, U^{T}\right)^{T}$,

$$
J_{2 N}=\left(\begin{array}{cc}
0 & I_{N} \\
-I_{N} & 0
\end{array}\right) .
$$

The semi-discrete system (2.2) obeys the symplectic conservation law

$$
\frac{d}{d t}\left(\frac{1}{2} d Z \wedge J_{2 N} d Z\right)=0
$$

where $d Z \wedge J_{2 N} d Z / 2$ is the symplectic structure of the system.

Remark 2.2. By applying the Fourier pseudospectral method to the Hamiltonian system (1.2), we obtain the semi-dicretization system which is a canonical Hamiltonian system.

\subsection{Full discretization in time}

In this subsection, we first propose a new symplectic Euler Fourier pseudospectral scheme (SE-FPS) for the KGS equation. By discretizing (2.3) in time by the symplectic Euler rule we have

$$
\Phi_{\tau}: \delta_{t}^{+} Z_{j}^{n}=J_{2 N}^{-1} \nabla H\left(Q_{j}^{n+1}, U_{j}^{n+1}, P_{j}^{n}, V_{j}^{n}\right),
$$

where $\delta_{t}^{+} Z_{j}^{n}=\left(Z_{j}^{n+1}-Z_{j}^{n}\right) / \tau$ is the forward difference operator and $\tau>0$ is the time step.

So we can obtain

$$
\begin{aligned}
& \frac{1}{\tau}\left(Q^{n+1}-Q^{n}\right)=-\frac{1}{2} D_{2} P^{n}-U^{n+1} \cdot P^{n}, \\
& \frac{1}{\tau}\left(U^{n+1}-U^{n}\right)=2 V^{n}, \\
& \frac{1}{\tau}\left(P^{n+1}-P^{n}\right)=\frac{1}{2} D_{2} Q^{n+1}+U^{n+1} \cdot Q^{n+1}, \\
& \frac{1}{\tau}\left(V^{n+1}-V^{n}\right)=\frac{1}{2}\left[D_{2} U^{n+1}-U^{n+1}+\left(P^{n}\right)^{\cdot 2}+\left(Q^{n+1}\right)^{\cdot 2}\right],
\end{aligned}
$$

which is called the symplectic Euler Fourier pseudospectral scheme (SE-FPS-1) for the KGS equation. 
Remark 2.3. From the expressions (2.5a)-(2.5d), it is observed that we can update $U^{n+1}$ by (2.5b), $V^{n+1}$ by (2.5d), $Q^{n+1}$ by $(2.5 \mathrm{a})$ and $P^{n+1}$ by (2.5c) respectively at every step. That is, this new scheme is explicit in nature.

It can be verified that accuracy for the new scheme is of spectral convergence rate in space and of one order in time.

In the same way we can construct another symplectic Euler Fourier pseudospectral scheme (SE-FPS-2)

$$
\Phi_{\tau}^{*}: \delta_{t}^{+} Z_{j}^{n}=J_{2 N}^{-1} \nabla H\left(Q^{n}, U^{n}, P^{n+1}, V^{n+1}\right),
$$

i.e.,

$$
\begin{aligned}
& \frac{1}{\tau}\left(Q^{n+1}-Q^{n}\right)=-\frac{1}{2} D_{2} P^{n+1}-U^{n} \cdot P^{n+1}, \\
& \frac{1}{\tau}\left(U^{n+1}-U^{n}\right)=2 V^{n+1}, \\
& \frac{1}{\tau}\left(P^{n+1}-P^{n}\right)=\frac{1}{2} D_{2} Q^{n}+U^{n} \cdot Q^{n}, \\
& \frac{1}{\tau}\left(V^{n+1}-V^{n}\right)=\frac{1}{2}\left[D_{2} U^{n}-U^{n}+\left(P^{n+1}\right)^{\cdot 2}+\left(Q^{n}\right)^{\cdot 2}\right] .
\end{aligned}
$$

The two new schemes have the same spatial discretization. We can prove that they are adjoint to each other in the temporal discretization. Similarly we can observe that the adjoint scheme is also explicit.

\subsection{Composition method}

Concept of the adjoint scheme can be found in lots of textbooks on numerical methods (see [8]).

The adjoint $\Phi_{\tau}^{*}$ of a method $\Phi_{\tau}$ is the inverse map of the original method with reversed time step $-\tau$, i.e., $\Phi_{\tau}^{*}=\Phi_{-\tau}^{-1}$. If $\Phi_{\tau}$ is a one step method of order $p$, the adjoint method $\Phi_{\tau}^{*}$ then has the same order $p$.

Therefore by apply the adjoint operation to the symplectic Euler Fourier pseudospectral scheme (2.4), we obtain its adjoint scheme (2.6). If the scheme $\Phi_{\tau}^{*}=\Phi_{\tau}$ we say it is symmetric. Furthermore if the method is symmetric, its (maximal) order is even.

By the composition theory for constructing high order scheme, we can composite the two schemes into a new explicit symplectic Fourier pseudospectral scheme (ES-FPS-1)

$$
\Psi_{\tau}^{[1]}=\Phi_{\frac{\tau}{2}}^{*} \circ \Phi_{\frac{\tau}{2}}
$$


as follows:

$$
\begin{aligned}
& \frac{2}{\tau}\left(Q^{n+\frac{1}{2}}-Q^{n}\right)=-\frac{1}{2} D_{2} P^{n}-U^{n+\frac{1}{2}} \cdot P^{n}, \\
& \frac{2}{\tau}\left(U^{n+\frac{1}{2}}-U^{n}\right)=2 V^{n}, \\
& \frac{2}{\tau}\left(P^{n+\frac{1}{2}}-P^{n}\right)=\frac{1}{2} D_{2} Q^{n+\frac{1}{2}}+U^{n+\frac{1}{2}} \cdot Q^{n+\frac{1}{2}}, \\
& \frac{2}{\tau}\left(V^{n+\frac{1}{2}}-V^{n}\right)=\frac{1}{2}\left[D_{2} U^{n+\frac{1}{2}}-U^{n+\frac{1}{2}}+\left(P^{n}\right)^{\cdot 2}+\left(Q^{n+\frac{1}{2}}\right)^{2}\right], \\
& \frac{2}{\tau}\left(Q^{n+1}-Q^{n+\frac{1}{2}}\right)=-\frac{1}{2} D_{2} P^{n+1}-U^{n+\frac{1}{2}} \cdot P^{n+1}, \\
& \frac{2}{\tau}\left(U^{n+1}-U^{n+\frac{1}{2}}\right)=2 V^{n+1}, \\
& \frac{2}{\tau}\left(P^{n+1}-P^{n+\frac{1}{2}}\right)=\frac{1}{2} D_{2} Q^{n+\frac{1}{2}}+U^{n+\frac{1}{2}} \cdot Q^{n+\frac{1}{2}}=\frac{2}{\tau}\left(P^{n+\frac{1}{2}}-P^{n}\right), \\
& \frac{2}{\tau}\left(V^{n+1}-V^{n+\frac{1}{2}}\right)=\frac{1}{2}\left[D_{2} U^{n+\frac{1}{2}}-U^{n+\frac{1}{2}}+\left(P^{n+1}\right)^{\cdot 2}+\left(Q^{n+\frac{1}{2}}\right)^{\cdot 2}\right] .
\end{aligned}
$$

Another composition scheme (ES-FPS-2)

$$
\Psi_{\tau}^{[2]}=\Phi_{\frac{\tau}{2}} \circ \Phi_{\frac{\tau}{2}}^{*}
$$

is

$$
\begin{aligned}
& \frac{2}{\tau}\left(Q^{n+\frac{1}{2}}-Q^{n}\right)=-\frac{1}{2} D_{2} P^{n+\frac{1}{2}}-U^{n} \cdot P^{n+\frac{1}{2}}, \\
& \frac{2}{\tau}\left(U^{n+\frac{1}{2}}-U^{n}\right)=2 V^{n+\frac{1}{2}}, \\
& \frac{2}{\tau}\left(P^{n+\frac{1}{2}}-P^{n}\right)=\frac{1}{2} D_{2} Q^{n}+U^{n} \cdot Q^{n}, \\
& \frac{2}{\tau}\left(V^{n+\frac{1}{2}}-V^{n}\right)=\frac{1}{2}\left[D_{2} U^{n}-U^{n}+\left(P^{n+\frac{1}{2}}\right)^{\cdot 2}+\left(Q^{n}\right)^{\cdot 2}\right], \\
& \frac{2}{\tau}\left(Q^{n+1}-Q^{n+\frac{1}{2}}\right)=-\frac{1}{2} D_{2} P^{n+\frac{1}{2}}-U^{n+1} \cdot P^{n+\frac{1}{2}}, \\
& \frac{2}{\tau}\left(U^{n+1}-U^{n+\frac{1}{2}}\right) U^{n+1}=2 V^{n+\frac{1}{2}}=\frac{2}{\tau}\left(U^{n+\frac{1}{2}}-U^{n}\right), \\
& \frac{2}{\tau}\left(P^{n+1}-P^{n+\frac{1}{2}}\right)=\frac{1}{2} D_{2} Q^{n+1}+U^{n+1} \cdot Q^{n+1}, \\
& \frac{2}{\tau}\left(V^{n+1}-V^{n+\frac{1}{2}}\right)=\frac{1}{2}\left[D_{2} U^{n+1}-U^{n+1}+\left(P^{n+\frac{1}{2}}\right)^{\cdot 2}+\left(Q^{n+1}\right)^{\cdot 2}\right] .
\end{aligned}
$$

We can see that the above scheme $\Psi_{\tau}^{[1]}$ is the composition of the symplectic Euler $\Phi_{\tau}^{*}$ with $\Phi_{\tau}$, while the scheme $\Psi_{\tau}^{[2]}$ is the composition of method $\Phi_{\tau}$ followed by $\Phi_{\tau}^{*}$. So $\Psi_{\tau}^{[2]}$ is also the adjoint of $\Psi_{\tau}^{[1]}$. Thus the two schemes are symmetric and totally explicit with accuracy of order two in temporal direction. 


\section{Numerical analysis of the scheme}

\subsection{Structure-Preserving properties}

Theorem 3.1. The two schemes $\Psi_{\tau}^{[1]}=\Phi_{\frac{\tau}{2}}^{*} \circ \Phi_{\frac{\tau}{2}}$ and $\Psi_{\tau}^{[2]}=\Phi_{\frac{\tau}{2}} \circ \Phi_{\frac{\tau}{2}}^{*}$ preserve the symplectic structure, i.e.,

$$
\delta_{t}^{+}\left(\frac{1}{2} d P^{n} \wedge d Q^{n}+\frac{1}{2} d V^{n} \wedge d U^{n}\right)=0 .
$$

Proof. Because the symplectic Euler scheme for canonical Hamiltonian systems is symplectic, the Euler Fourier pseudospectral method (2.4) $\Phi_{\tau}$ and its adjoint method (2.5) $\Phi_{\tau}^{*}$ preserve the same symplectic structure, i.e.,

$$
\delta_{t}^{+}\left(\frac{1}{2} d P^{n} \wedge d Q^{n}+\frac{1}{2} d V^{n} \wedge d U^{n}\right)=0 .
$$

By the theory of composition we can know that both the two schemes $\Psi_{\tau}^{[1]}$ and $\Psi_{\tau}^{[2]}$ are symplectic.

\subsection{Linear stability analysis}

To show the stability of the numerical method, i.e., the sensitivity of the numerical solution to perturbations in the initial data, the Von-Neumann stability is analyzed.

First we remove the nonlinear term and rewrite the symplectic Euler scheme (2.5a)(2.5d) in the matrix form

$$
\left(\begin{array}{cccc}
I & 0 & 0 & 0 \\
0 & I & 0 & 0 \\
-\frac{\tau}{2} D_{2} & 0 & I & 0 \\
0 & -\frac{\tau}{2}\left(D_{2}-I\right) & 0 & I
\end{array}\right)\left(\begin{array}{c}
Q^{n+1} \\
U^{n+1} \\
P^{n+1} \\
V^{n+1}
\end{array}\right)=\left(\begin{array}{cccc}
I & 0 & -\frac{\tau}{2} D_{2} & 0 \\
0 & I & 0 & 2 \tau I \\
0 & 0 & I & 0 \\
0 & 0 & 0 & I
\end{array}\right)\left(\begin{array}{c}
Q^{n} \\
U^{n} \\
P^{n} \\
V^{n}
\end{array}\right)
$$

The amplification matrix of (3.1) is

$$
\begin{aligned}
M_{1} & =\left(\begin{array}{cccc}
I & 0 & 0 & 0 \\
0 & I & 0 & 0 \\
-\frac{\tau}{2} D_{2} & 0 & I & 0 \\
0 & -\frac{\tau}{2}\left(D_{2}-I\right) & 0 & I
\end{array}\right)^{-1}\left(\begin{array}{cccc}
I & 0 & -\frac{\tau}{2} D_{2} & 0 \\
0 & I & 0 & 2 \tau I \\
0 & 0 & I & 0 \\
0 & 0 & 0 & I
\end{array}\right) \\
& =\left(\begin{array}{cccc}
I & 0 & -\frac{\tau}{2} D_{2} & 0 \\
0 & I & 0 & 2 \tau I \\
\frac{\tau}{2} D_{2} & 0 & I-\frac{\tau^{2}}{4} D_{2}^{2} & 0 \\
0 & \frac{\tau}{2}\left(D_{2}-I\right) & 0 & I+\tau^{2}\left(D_{2}-I\right)
\end{array}\right) .
\end{aligned}
$$


According to (2.1), the eigenvalues of $M_{1}$ satisfy

$$
\lambda^{2}+\left(\frac{\tau^{2}}{4} \lambda_{k}^{2}-2\right) \lambda+1=0, \quad \text { or } \quad \lambda^{2}-\left[\tau^{2}\left(\lambda_{k}-1\right)+2\right] \lambda+1=0,
$$

where

$$
\lambda_{k}= \begin{cases}-(k \mu)^{2}, & k=0,1, \cdots, N / 2 \\ -[(k-N) \mu]^{2}, & k=N / 2+1, \cdots, N-1 .\end{cases}
$$

So

$$
|\lambda| \leq 1
$$

if and only if

$$
\left|\frac{\tau^{2}}{4} \lambda_{k}^{2}-2\right| \leq 2, \quad\left|\tau^{2}\left(\lambda_{k}-1\right)+2\right| \leq 2
$$

Similarly, the stability analysis of the symplectic Euler scheme (2.7) has the same results. The matrix form of the scheme is

$$
\left(\begin{array}{cccc}
I & 0 & \frac{\tau}{2} D_{2} & 0 \\
0 & I & 0 & -2 \tau I \\
0 & 0 & I & 0 \\
0 & 0 & 0 & I
\end{array}\right)\left(\begin{array}{c}
Q^{n+1} \\
U^{n+1} \\
P^{n+1} \\
V^{n+1}
\end{array}\right)=\left(\begin{array}{cccc}
I & 0 & 0 & 0 \\
0 & I & 0 & 0 \\
\frac{\tau}{2} D_{2} & 0 & I & 0 \\
0 & \frac{\tau}{2}\left(D_{2}-I\right) & 0 & I
\end{array}\right)\left(\begin{array}{c}
Q^{n} \\
U^{n} \\
P^{n} \\
V^{n}
\end{array}\right)
$$

The amplification matrix of (3.3) is

$$
M_{2}=\left(\begin{array}{cccc}
I-\frac{\tau^{2}}{4} D_{2}^{2} & 0 & -\frac{\tau}{2} D_{2} & 0 \\
0 & I+\tau^{2}\left(D_{2}-I\right) & 0 & 2 \tau I \\
\frac{\tau}{2} D_{2} & 0 & I & 0 \\
0 & \frac{\tau}{2}\left(D_{2}-I\right) & 0 & I
\end{array}\right) .
$$

The eigenvalues of $M_{2}$ satisfy $|\lambda| \leq 1$ if and only if

$$
\left|\frac{\tau^{2}}{4} \lambda_{k}^{2}-2\right| \leq 2, \quad\left|\tau^{2}\left(\lambda_{k}-1\right)+2\right| \leq 2
$$

From (3.2) and (3.4), we can obtain the following theorem.

Theorem 3.2. The necessary and sufficient conditions for the stability of the symplectic Euler Fourier pseudospectral method $\Phi_{\tau}$ and $\Phi_{\tau}^{*}$ are

$$
\frac{\tau}{h^{2}} \leq \frac{4}{\pi^{2}} \simeq 0.405, \quad \frac{\tau^{2}}{h} \leq \frac{2}{\pi} \simeq 0.637
$$


Now we study the stability of the new explicit symplectic methods (2.8) and (2.9). The amplification matrix of $\Psi_{\tau}^{[1]}$ is

$$
M=M_{2} M_{1}=\left(\begin{array}{cccc}
I-\frac{\tau^{2}}{8} D_{2}^{2} & 0 & -\frac{\tau}{2} D_{2}+\frac{\tau^{3}}{32} D_{2}^{3} & 0 \\
0 & I+\frac{\tau^{2}}{2}\left(D_{2}-I\right) & 0 & 2 \tau I+\frac{\tau^{3}}{2}\left(D_{2}-I\right) \\
\frac{\tau}{2} D_{2} & 0 & I-\frac{\tau^{2}}{8} D_{2}^{2} & 0 \\
0 & \frac{\tau}{2}\left(D_{2}-I\right) & 0 & I+\frac{\tau^{2}}{2}\left(D_{2}-I\right)
\end{array}\right) .
$$

The amplification matrix of $\Psi_{\tau}^{[2]}$ is $\tilde{M}=M_{1} M_{2}$ which have the same eigenvalues with $M$. Since

$$
\begin{aligned}
|\lambda I-M|= & \prod_{k=0}^{N-1}\left[\left(\lambda-1+\frac{\tau^{2}}{8} \lambda_{k}^{2}\right)^{2}-\frac{\tau}{2} \lambda_{k}\left(-\frac{\tau}{2} \lambda_{k}+\frac{\tau^{3}}{32} \lambda_{k}^{3}\right)\right] \\
& \times\left[\left(\lambda-1+\frac{\tau^{2}}{2}\left(\lambda_{k}-1\right)\right)^{2}-\frac{\tau}{2}\left(\lambda_{k}-1\right)\left(2 \tau+\frac{\tau^{3}}{2}\left(\lambda_{k}-1\right)\right)\right] \\
= & \prod_{k=0}^{N-1}\left[\lambda^{2}+\left(\frac{\tau^{2}}{4} \lambda_{k}^{2}-2\right) \lambda+1\right]\left[\lambda^{2}+\left(-\tau^{2}\left(\lambda_{k}-1\right)-2\right) \lambda+1\right] .
\end{aligned}
$$

So we can conclude that the two new schemes have the same linear stability with symplectic Euler Fourier pseudospectral method.

Theorem 3.3. The new second-order explicit symplectic Fourier pseudospectral methods (ESFPS) (2.8) and (2.9) have the linear stability if and only if

$$
\frac{\tau}{h^{2}} \leq \frac{4}{\pi^{2}} \simeq 0.405, \quad \frac{\tau^{2}}{h} \leq \frac{2}{\pi} \simeq 0.637 .
$$

The CFL stability condition is confirmed by the numerical examples in Section 4 .

\section{Numerical examples}

In this section, some numerical experiments are carried out to show the performance of the new explicit symplectic methods. The performance of the proposed method will be exhibited in following aspects:

(i) to test convergence order in the temporal direction and the spatial direction respectively;

(ii) to check the linear stability of the schemes; 
(iii) to verify the ability in preserving the symplectic structure.

Accuracy of migration of soliton at $t_{n}=n \tau$ is measured by

$$
\|\varphi\|_{L_{\infty}}=\max _{1 \leq j \leq N}\left|\varphi\left(x_{j}, t_{n}\right)-\varphi_{j}^{n}\right|
$$

and

$$
\|\varphi\|_{L_{2}}=\left(h \sum_{j=1}^{N}\left|\varphi\left(x_{j}, t_{n}\right)-\varphi_{j}^{n}\right|^{2}\right)^{1 / 2} .
$$

The relative energy error and relative mass error at $t=t_{n}$ are defined as

$$
R H_{n}=\frac{\left|H^{n}-H^{0}\right|}{\left|H^{0}\right|}, \quad R M_{n}=\frac{\left|M^{n}-M^{0}\right|}{\left|M^{0}\right|}
$$

where

$$
\begin{aligned}
& H^{n}=H\left(P^{n}, V^{n}, Q^{n}, U^{n}\right), \\
& M^{n}=M\left(P^{n}, V^{n}, Q^{n}, U^{n}\right), \\
& H(Z)=-\frac{1}{4}\left(P^{T} D_{2} P+Q^{T} D_{2} Q+U^{T} D_{2} U\right)+\frac{1}{4} U^{T} U+V^{T} V-\frac{1}{2} \sum_{j=0}^{N-1}\left(p_{i}^{2}+q_{j}^{2}\right) u_{j} \\
& M(Z)=P^{T} P+Q^{T} Q
\end{aligned}
$$

and $H(Z)$ and $M(Z)$ denote the energy and the mass at $t=t_{n}, n=0,1, \cdots$, respectively.

The discrete $L^{2}$-error and the discrete $L^{\infty}$-error at $t=t_{n}$ are defined as

$$
\begin{aligned}
& L^{2}-\operatorname{error}_{n}(N, \tau)=\left\|z^{n}-Z^{n}\right\|_{2} \\
& =\left(\left\|p^{n}-P^{n}\right\|_{2}^{2}+\left\|v^{n}-V^{n}\right\|_{2}^{2}+\left\|q^{n}-Q^{n}\right\|_{2}^{2}+\left\|u^{n}-U^{n}\right\|_{2}^{2}\right)^{1 / 2}, \\
& \begin{aligned}
L^{\infty}-\operatorname{error}_{n}(N, \tau) & =\left\|z^{n}-Z^{n}\right\|_{\infty} \\
& =\max \left\{\left\|p^{n}-P^{n}\right\|_{\infty},\left\|v^{n}-V^{n}\right\|_{\infty},\left\|q^{n}-Q^{n}\right\|_{\infty},\left\|u^{n}-U^{n}\right\|_{\infty}\right\},
\end{aligned}
\end{aligned}
$$

where $h=L / N$ is the space step and $\tau$ is the time step, and

$$
\begin{aligned}
& p^{n}=\left(p\left(x_{0}, t_{n}\right), p\left(x_{1}, t_{n}\right), \cdots, p\left(x_{N-1}, t_{n}\right)\right)^{T}, \\
& P^{n}=\left(p_{0}^{n}, p_{1}^{n}, \cdots, p_{N-1}^{n}\right)^{T}, \\
& \left\|p^{n}-P^{n}\right\|_{2}=\left(h \sum_{j=0}^{N-1}\left|p\left(x_{j}, t_{n}\right)-p_{j}^{n}\right|^{2}\right)^{1 / 2}, \\
& \left\|p^{n}-P^{n}\right\|_{\infty}=\max _{0 \leq j \leq N-1}\left|p\left(x_{j}, t_{n}\right)-p_{j}^{n}\right| .
\end{aligned}
$$


For fixed $\tau_{0}$ and $N_{0}$ we define the space and time order of the method

$$
\operatorname{Order}_{N}\left(\tau_{0}\right)=-\frac{\log _{2}\left(\frac{\operatorname{error}_{n}\left(N_{1}, \tau_{0}\right)}{\operatorname{error}_{n}\left(N_{2}, \tau_{0}\right)}\right)}{\log _{2}\left(\frac{N_{1}}{N_{2}}\right)}, \quad \operatorname{Order}_{\tau}\left(N_{0}\right)=\frac{\log _{2}\left(\frac{\operatorname{error}_{n}\left(N_{0}, \tau_{1}\right)}{\operatorname{error}_{n}\left(N_{0}, \tau_{2}\right)}\right)}{\log _{2}\left(\frac{\tau_{1}}{\tau_{2}}\right)} .
$$

From [20], the KGS equation has the soliton solution

$$
\begin{aligned}
& \varphi\left(x, t, v, x_{0}\right)=\frac{3 \sqrt{2}}{4 \sqrt{1-v^{2}}} \operatorname{sech}^{2}\left(\frac{1}{2 \sqrt{1-v^{2}}}\left(x-v t-x_{0}\right)\right) \exp \left(i\left(v x+\frac{1-v^{2}+v^{4}}{2\left(1-v^{2}\right)} t\right)\right), \\
& u\left(x, t, v, x_{0}\right)=\frac{3}{4\left(1-v^{2}\right)} \operatorname{sech}^{2}\left(\frac{1}{2 \sqrt{1-v^{2}}}\left(x-v t-x_{0}\right)\right) .
\end{aligned}
$$

Here, $|v|<1$ represents the propagating velocity of the wave and $x_{0}=0$ is the initial phase. We can see that $\varphi(x, t)$ and $u(x, t)$ decay to zero as $|x| \rightarrow \infty$ for a fixed $t$, so numerically we can solve the KGS equation in a finite domain. Now we carry out three numerical experiments to test the theoretical analysis on the difference solutions.

Example 4.1 (Single soliton). We consider the KGS equation with initial conditions

$$
\begin{aligned}
& \varphi\left(x, 0, v, x_{0}\right)=\frac{3 \sqrt{2}}{4 \sqrt{1-v^{2}}} \operatorname{sech}^{2}\left(\frac{1}{2 \sqrt{1-v^{2}}}\left(x-x_{0}\right)\right) \exp (i v x), \\
& u\left(x, 0, v, x_{0}\right)=\frac{3}{4\left(1-v^{2}\right)} \operatorname{sech}^{2}\left(\frac{1}{2 \sqrt{1-v^{2}}}\left(x-x_{0}\right)\right), \\
& u_{t}\left(x, 0, v, x_{0}\right)=\frac{3 v}{4\left(1-v^{2}\right)^{\frac{3}{2}}} \operatorname{sech}^{2}\left(\frac{1}{2 \sqrt{1-v^{2}}}\left(x-x_{0}\right)\right) \tanh \left(\frac{1}{2 \sqrt{1-v^{2}}}\left(x-x_{0}\right)\right) .
\end{aligned}
$$

We choose $v=-0.8, \tau=0.01$ and $h=0.25$. The computations are done on the space interval $\Omega=[-20,20]$ with the periodic boundary conditions $\varphi(-20, t)=\varphi(20, t)$, $u(-20, t)=u(20, t), u_{t}(-20, t)=u_{t}(20, t)$ and different $N$ and $\tau$ to test error estimate. We test the the order of convergence in the temporal direction and the spatial direction respectively.

Firstly, we set $N=200$ to ignore the error in the spatial direction. Table 1 and Table 2 show the numerical results of the symplectic Euler and the composition scheme with different time steps from $t=0$ to $t=1$ respectively. It is easy to see that the convergence order of symplectic Euler method is almost equal to 1 in the temporal direction and composition method is almost equals to 2, which supports the theoretical analysis.

Secondly, we set $\tau=0.001$ to ignore the error in the temporal direction. Table 3 shows the numerical results of the symplectic Euler Fourier pseudospectral method with different $N$ from $t=0$ to $t=100$. The spectral accuracy of the method in space is demonstrated. We can conclude that the error estimate is confirmed very well. The results in Table 4 and Table 5 show the stability of the methods. We find that the scheme is unstable and the solution blows up as the mesh ratio does not satisfy the stability condition of Theorem 
Table 1: The numerical results of the symplectic Euler method with $N=200$ and different time steps.

\begin{tabular}{||ccccc||}
\hline$t_{n}=1$ & $L^{\infty}-\operatorname{error}_{n}(\tau)$ & $L^{\infty}-\operatorname{Order}_{\tau}$ & $L^{2}-\operatorname{error}_{n}(\tau)$ & $L^{2}-\operatorname{Order}_{\tau}$ \\
\hline$\tau_{1}=0.004$ & $8.938 \times 10^{-4}$ & - & $1.425 \times 10^{-3}$ & - \\
\hline$\tau_{2}=0.002$ & $4.474 \times 10^{-4}$ & 0.998 & $7.137 \times 10^{-4}$ & 0.998 \\
\hline$\tau_{3}=0.001$ & $2.238 \times 10^{-4}$ & 0.994 & $3.571 \times 10^{-4}$ & 0.999 \\
\hline$\tau_{4}=0.0005$ & $1.119 \times 10^{-4}$ & 1.000 & $1.786 \times 10^{-4}$ & 1.000 \\
\hline$\tau_{5}=0.00025$ & $5.598 \times 10^{-5}$ & 0.999 & $8.931 \times 10^{-5}$ & 0.999 \\
\hline
\end{tabular}

Table 2: The numerical results of the composition method with $N=200$ and different time steps.

\begin{tabular}{||ccccc||}
\hline$t_{n}=1$ & $L^{\infty}-\operatorname{error}_{n}(\tau)$ & $L^{\infty}-\operatorname{Order}_{\tau}$ & $L^{2}$-error $_{n}(\tau)$ & $L^{2}-$ Order $_{\tau}$ \\
\hline$\tau_{1}=0.004$ & $2.1448 \times 10^{-6}$ & - & $3.0739 \times 10^{-6}$ & - \\
\hline$\tau_{2}=0.002$ & $5.3618 \times 10^{-7}$ & 2.0000 & $7.6848 \times 10^{-7}$ & 1.99999 \\
\hline$\tau_{3}=0.001$ & $1.3403 \times 10^{-7}$ & 2.0002 & $1.9215 \times 10^{-7}$ & 1.9998 \\
\hline$\tau_{4}=0.0005$ & $3.3497 \times 10^{-8}$ & 2.0005 & $4.8151 \times 10^{-8}$ & 1.9966 \\
\hline$\tau_{5}=0.00025$ & $8.3632 \times 10^{-9}$ & 2.0019 & $1.2469 \times 10^{-8}$ & 1.9492 \\
\hline
\end{tabular}

Table 3: The numerical results of the composition method with $\tau=0.001$ and different $N$.

\begin{tabular}{||ccccc||}
\hline$\tau=0.001$ & $L^{\infty}$-error & $(N)$ & $L^{\infty}$-Order & $L^{2}$-error \\
$n$ & $(N)$ & $L^{2}$-Order $_{N}$ \\
\hline$N_{1}=128$ & $1.22 \times 10^{-4}$ & - & $1.88 \times 10^{-2}$ & - \\
\hline$N_{2}=132$ & $6.63 \times 10^{-5}$ & 14.12 & $9.22 \times 10^{-3}$ & 16.49 \\
\hline$N_{3}=136$ & $4.32 \times 10^{-5}$ & 14.38 & $5.54 \times 10^{-3}$ & 17.05 \\
\hline$N_{4}=142$ & $2.74 \times 10^{-5}$ & 14.79 & $3.24 \times 10^{-3}$ & 17.49 \\
\hline
\end{tabular}

Table 4: Stability of the symplectic Euler and the composition method with $\tau=0.01$ and different mesh ratios at $t=2$.

\begin{tabular}{||cccc||}
\hline$\tau=0.01$ & $\frac{\tau}{h^{2}}$ & symplectic Euler-error & composition-error \\
\hline$h_{1}=0.2$ & 0.25 & $6.71 \times 10^{-3}$ & $2.31 \times 10^{-5}$ \\
\hline$h_{2}=0.25$ & 0.16 & $5.90 \times 10^{-3}$ & $2.06 \times 10^{-5}$ \\
\hline$h_{3}=0.166$ & 0.36 & $5.54 \times 10^{-3}$ & $1.88 \times 10^{-5}$ \\
\hline$h_{4}=0.16$ & 0.39 & $5.31 \times 10^{-3}$ & $1.84 \times 10^{-5}$ \\
\hline$h_{4}=0.143$ & 0.49 & blow up & blow up \\
\hline$h_{4}=0.125$ & 0.64 & blow up & blow up \\
\hline
\end{tabular}

Table 5: Stability of the symplectic Euler with different mesh ratios $\frac{\tau}{h^{2}}$.

\begin{tabular}{||cccccc||}
\hline & $\tau_{1}=0.01$ & $\tau_{2}=0.02$ & $\tau_{3}=0.03$ & $\tau_{4}=0.04$ & $\tau_{5}=0.05$ \\
\hline$N_{1}=100$ & 0.625 & 0.125 & 0.1875 & 0.25 & 0.3125 \\
\hline$N_{2}=120$ & 0.09 & 0.18 & 0.27 & 0.36 & 0.45 (blow up) \\
\hline$N_{3}=140$ & 0.1225 & 0.245 & 0.37 & 0.49 (blow up) & 0.61 (blow up) \\
\hline$N_{4}=150$ & 0.14 & 0.28 & 0.42 (blow up) & 0.56 (blow up) & 0.70 (blow up) \\
\hline$N_{4}=160$ & 0.125 & 0.32 & 0.48 (blow up) & 0.64 (blow up) & 0.80 (blow up) \\
\hline
\end{tabular}




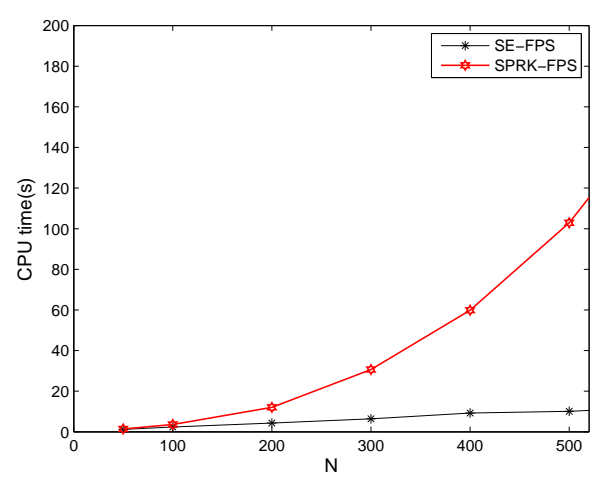

(a)

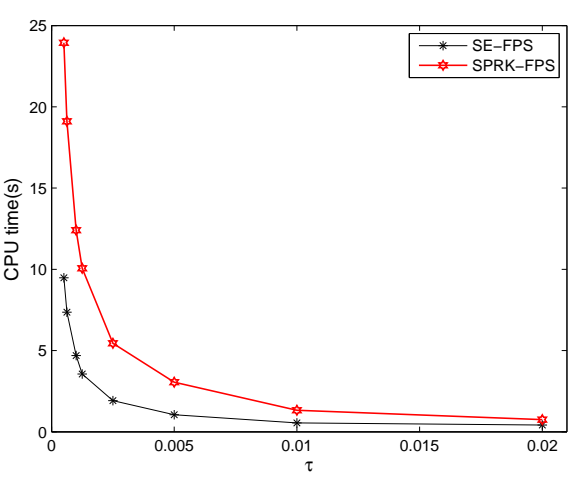

(b)

Figure 1: The CPU time for SE-FPS and SPRK-FPS methods with different $N$ and $\tau$. (a) CPU time from $t=0$ to $t=1$ when $\tau=0.001$. (b) CPU time from $t=0$ to $t=1$ when $N=200$.

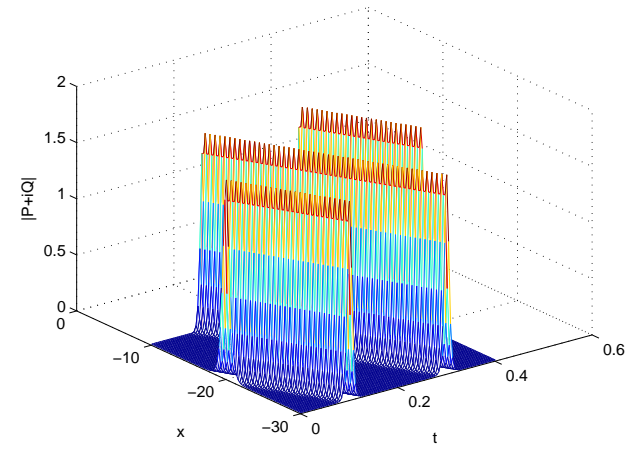

(a)

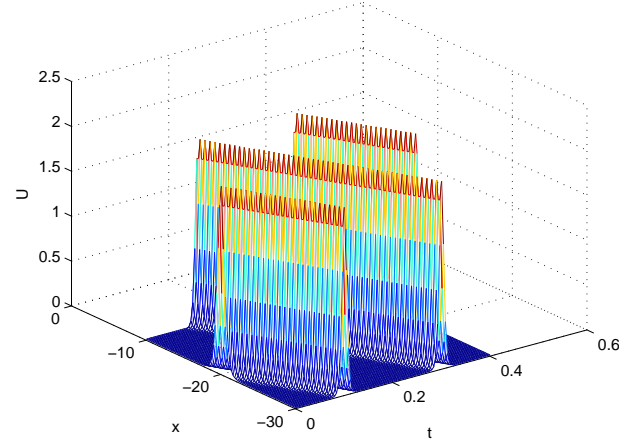

(b)

Figure 2: The numerical solution of the symplectic Euler Fourier pseudo-spectral method solving the Example 4.1. (a) Propagation of solitary wave from $t=0$ to $t=1$ of $\varphi(x, t)$. (b) Propagation of solitary wave from $t=0$ to $t=1$ of $u(x, t)$.

3.3, which agrees with the theoretical analysis. Under the same mesh partition, the symplectic Euler and the composition method have the same stability but the latter is more effective and more rapid than the former.

Thirdly, in order to make a comparison of the computational effectiveness of our new explicit symplectic methods (SE-FPS) and other symplectic method, we measure the CPU time of SE-FPS method and SPRK-FPS method in [12] under different mesh generation for the equation from $t=0$ to $t=1$. Fig. 1 shows the CPU time in double logarithmic scales. Obviously, the SE-FPS scheme is more effective than the SPRK-FPS scheme.

Finally, Fig. 2 shows the shapes of soliton $\varphi(x, t)$ and $u(x, t)$ from $t=0$ to $t=100$. Fig. 3 shows the shapes of soliton $\varphi(x, t)$ and $u(x, t)$ from $t=0$ to $t=1050$. The values of energy and mass in discrete level are listed in Fig. 4. Although our new scheme can not conserve 


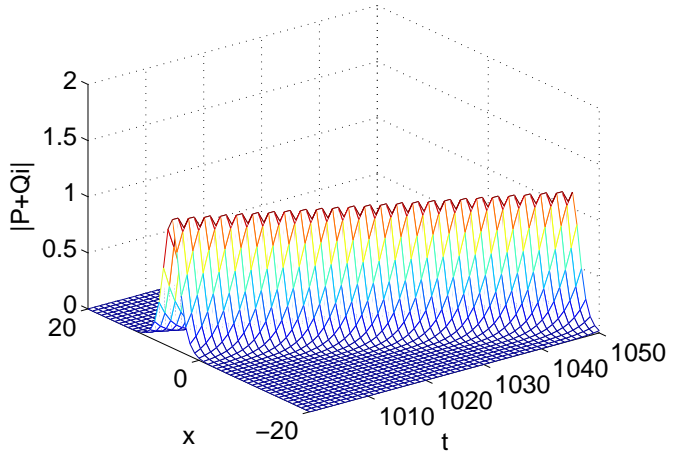

(a)

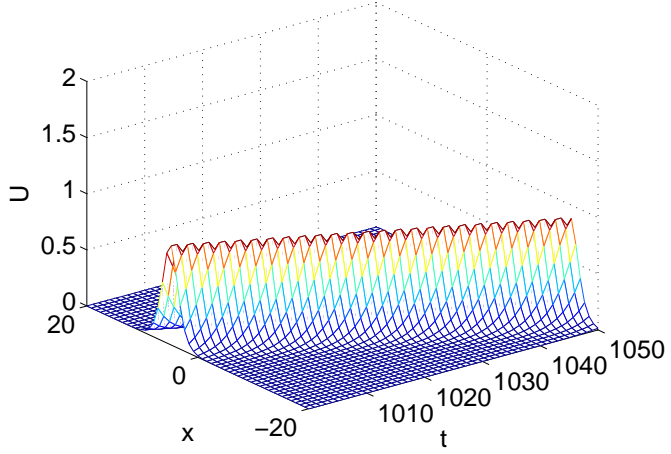

(b)

Figure 3: (a) Propagation of solitary wave from from $t=0$ to $t=1050$ of $\varphi(x, t)$. (b) Propagation of solitary wave from $t=0$ to $t=1050$ of $u(x, t)$.

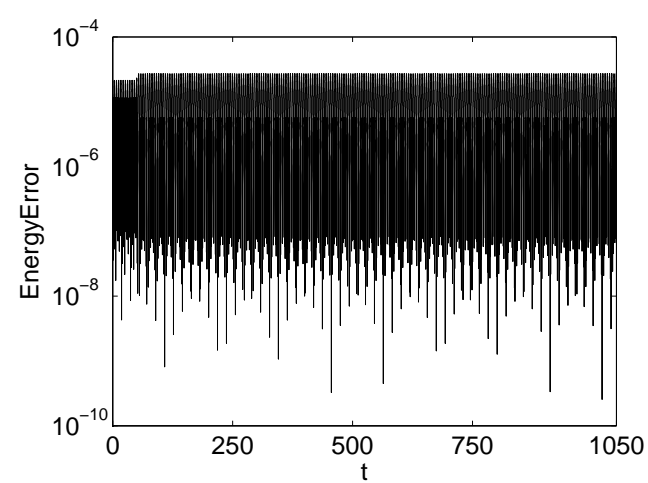

(a)

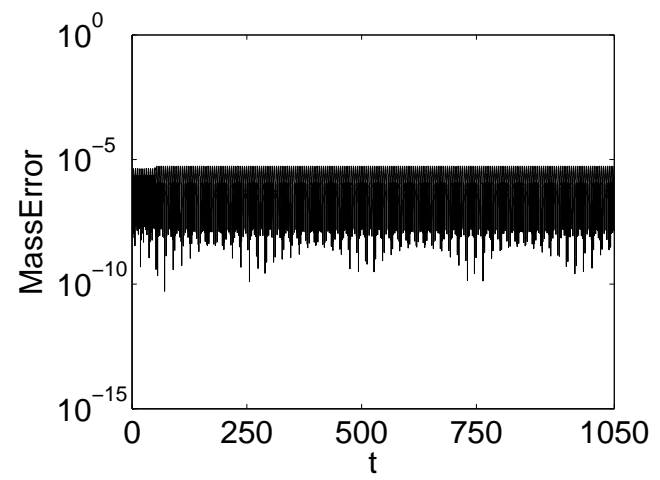

(b)

Figure 4: The numerical solution of the composition method solving the Example 4.1. (a) Relative energy error of propagation of solitary wave from $t=0$ to $t=1050$. (b) Relative mass error of propagation of solitary wave from $t=0$ to $t=1050$.

discrete mass and energy conservation exactly, it is observed from Fig. 4 that their errors are very small during longtime simulation, which demonstrate the superior behavior of the symplectic scheme.

Example 4.2 (Solitons collision). In the following simulations, we will study head-on collisions of two solitary waves. The initial conditions are chosen as

$$
\begin{aligned}
& \varphi_{0}=\varphi\left(x, 0, v_{1}, x_{1}\right)+\varphi\left(x, 0, v_{2}, x_{2}\right), \\
& u_{0}=u\left(x, 0, v_{1}, x_{1}\right)+u\left(x, 0, v_{2}, x_{2}\right), \\
& u_{1}=u_{t}\left(x, 0, v_{1}, x_{1}\right)+u_{t}\left(x, 0, v_{2}, x_{2}\right) .
\end{aligned}
$$




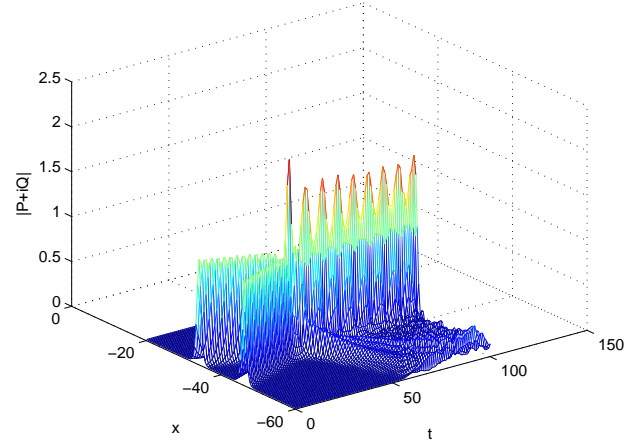

(a)

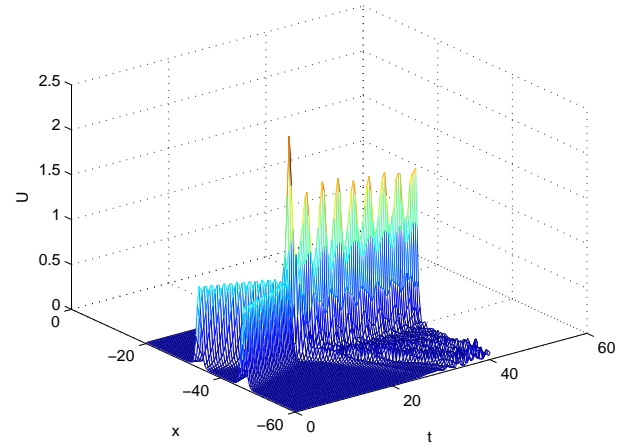

(b)

Figure 5: The numerical solution of the composition method solving the Example 4.2. (a) Symmetric solitons collision from $t=0$ to $t=100$ of $\varphi(x, t)$. (b) Propagation of solitary wave from $t=0$ to $t=100$ of $u(x, t)$.

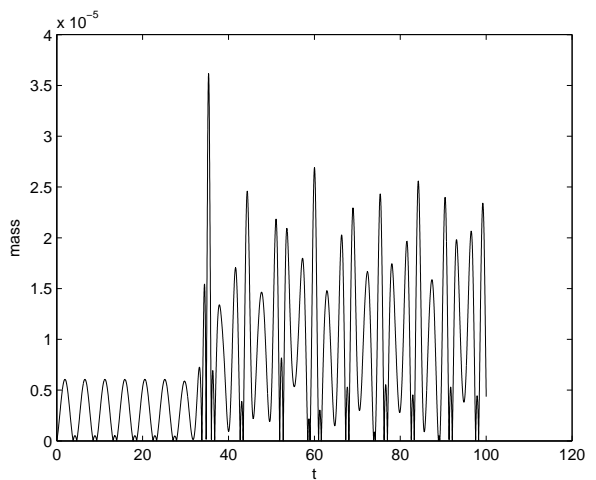

(a)

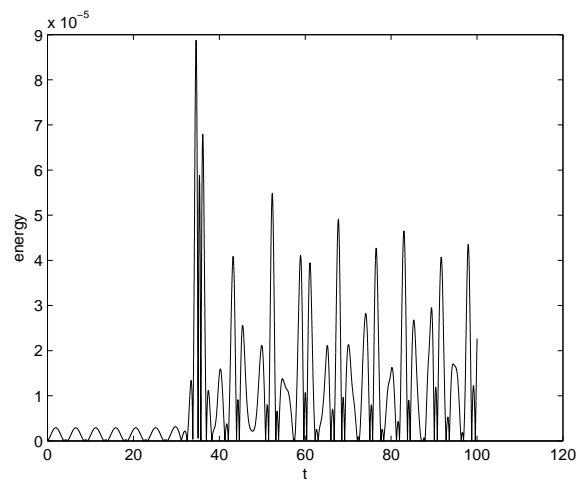

(b)

Figure 6: The numerical solution of the composition method solving the Example 4.2. (a) Relative mass error of propagation of solitary wave from $t=0$ to $t=100$. (b) Relative energy error of propagation of solitary wave from $t=0$ to $t=100$.

We investigate the collision of symmetric solitons, i.e., $v_{1}=0.4, x_{1}=-15$ and $v_{2}=-0.4$, $x_{2}=15$ over the region $[-50,50] \times[0,100]$ with parameters $\tau=0.01$ and $h=0.25$. Fig. 5 displays the evolution of the shapes of $\phi$ and $u$.

We can find that the two solitons stay at the same position at the time around $t=25$ and the solitons keep in this state. After that the collision results in fusion accompanied by the series of emissions of waves. Fig. 6 displays the energy and mass.

\section{Conclusions}

Based on symplectic Euler method, Fourier pseudo-spectral method and composition technique, we have introduced a new explicit symplectic scheme to simulate the KGS 
equation. It is noteworthy that this scheme is a totally explicit scheme of second order in time and spectral accuracy in space. This means that it dose not need to solve any nonlinear algebraic equation at each time step. In this sense our scheme is more favorable to improve the efficiency of simulation than the general implicit symplectic schemes and non-symplectic ones. Besides, its relative linear stability is analyzed in detail.

Numerical results indicate that the proposed scheme can provide numerical solutions and also show excellent performance in preserving invariants of the system in long-term computing.

\section{Acknowledgements}

This work is supported by the Jiangsu Collaborative Innovation Center for Climate Change, the National Natural Science Foundation of China (Grant Nos. 11271195 and 11271196) and the Priority Academic Program Development of Jiangsu Higher Education Institutions.

\section{References}

[1] W. BAO AND L. YANG, Efficient and accurate numerical methods for the Klein-GordonSchrödinger equations, J. Comput. Phys., 225 (2007), pp. 1863-1893.

[2] T. J. BRIDGES, Multi-symplectic structures and wave propagation, Math. Proc. Camb. Phil. Soc., 121 (1997), pp. 147-190.

[3] T. J. BRIDGES AND S. REICH, Multi-symplectic Integrators: numerical schemes for Hamiltonian PDEs that conserve symplecticity, Phys. Lett., 284 (2001), pp. 184-193.

[4] J. CHEN AND M. QIN, Multi-symplectic Fourier pseudospectral method for the nonlinear Schrödinger equation, Electronic Trans. Numer. Anal., 12 (2001), pp. 193-204.

[5] K. FENG, Difference schemes for Hamiltonian formalism and symplectic geometry, J. Comput. Math., 4 (1986), pp. 279-289.

[6] I. FUKUDA AND M. TSUTSUMI, On the Yukawa coupled Klein-Gordon-Schrödinger equations in the three space dimension, Proc. JPN. Acad., 51 (1975), pp. 402-405.

[7] B. GUO AND C. MIAO, Global existence and asymptotic behavior of the solution for the KleinGordon-Schrödinger equations, Sci. China, Ser. A, 25 (1995), pp. 705-714.

[8] E. Hairer, C. Lubich AND G. WANNER, Geometric Numerical Integration: Structure Preserving Algorithms for Ordinary Differential Equations, Berlin: Springer-Verlag, 2002.

[9] J. HONG, S. JIANG AND C. LI, Explicit multi-symplectic method for the Klein-GordonSchrödinger equations, J. Comput. Phys., 228 (2009), pp. 3517-3532.

[10] J. HONG, S. JIANG, L. KONG AND C. LI, Numerical comparison of five difference schemes for coupled Klein-Gordon-Schrödinger equations in quautum physics, J. Phys. A Math. Theor., 40 (2007), pp. 9125-9135.

[11] M. S. ISMAIL AND T. R. TAHA, A linearly implicit conservative scheme for the coupled nonliear Schrödinger equation, Math. Comput. Simul., 74 (2007), pp. 302-311.

[12] L. KOnG, J. ZHANG, Y. CAO, Y. DUAN AND H. HuANG, Semi-explicit symplectic partitioned Runge-Kutta Fourier Pseudo-spectral scheme for the Klein-Gordon-Schrödinger equations, Comput. Phys. Commun., 181 (2010), pp. 1369-1377. 
[13] R. MClachlan, Symplectic integration of Hamiltonian wave equations, Numer. Math., 66 (1993), pp. 465-492.

[14] T. Ozawa And Y. Tsutsumi, Asymptotic behavior of solution for the coupled Klein-GordonSchrödinger equations, Adv. Stu. Pure Math., 23 (1994), pp. 295-305.

[15] M. QIN AND M. ZHANG, Multi-stage symplectic schemes of two kinds of Hamiltonian systems for wave equations, J. Comput. Math. Appl., 4 (1990), pp. 51-62.

[16] S. REICH, Multi-symplectic Runge-Kutta collocation methods for Hamiltonian wave equations, J. Comput. Phys., 157 (2000), pp. 473-499.

[17] J. Shen AND T. TANG, Spectral and High-order Methods with Applications, Science Press, 2006.

[18] T. WANG, Optimal point-wise error estimate of a compact difference scheme for the Klein-GordonSchrödinger equations, J. Math. Anal. Appl., 412 (2014), pp. 155-167.

[19] Y. WANG, Q. LI AND Y. SONG, Two new simple multi-symplectic schemes for the nonlinear Schrödinger equation, China Phys. Lett., 25(5) (2008), pp. 1538-1540.

[20] J. XIA, Exact solitary solution of coupled Klein-Gordon-Schrödinger equations, Appl. Math. Mech., 23 (2002), pp. 52-57.

[21] L. ZHANG, Convergence of a conservative difference scheme for a class of Klein-Gordon-Schrödinger equations in one space dimension, Appl. Math. Comput., 163 (2005), pp. 343-355. 